\title{
Bonding Thin Mirror Segments without Distortion for the International X-Ray Observatory
}

\author{
Tyler C. Evans \\ 301-286-2677, tyler.evans@nasa.gov \\ Kai-Wing Chan \\ 301-286-6238, Kai-Wing.Chan-1@nasa.gov \\ Timo T. Saha \\ 301-286-2863, timo.t.saha@nasa.gov \\ NASA Goddard Space Flight Center \\ Greenbelt, MD 20771
}

\begin{abstract}
The International X-Ray Observatory (IXO) uses thin glass optics to maximize large effective area and precise low angular resolution. The thin glass mirror segments must be transferred from their fabricated state to a permanent structure without imparting distortion. IXO will incorporate about fourteen thousand thin mirror segments to achieve the mission goal of 3.0 square meters of effective area at $1.25 \mathrm{keV}$ with an angular resolution of five arcseconds. To preserve figure and alignment, the mirror segment must be bonded with sub-micron movement at each corner. Recent advances in technology development have produced significant $\mathrm{x}$-ray test results of a bonded pair of mirrors. Three specific bonding cycles will be described highlighting the improvements in procedure, temperature control, and precision bonding. This paper will highlight the recent advances in alignment and permanent bonding as well as the results they have produced. ${ }^{12}$
\end{abstract}

\section{TABLE OF CONTENTS}

1. INTRODUCTION

2. MIRROR SEGMENTS ..................................................................2

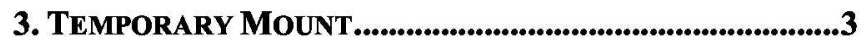

4. MIRROR ALIGNMENT ....................................................................3

5. VERIFICATION TESTING........................................................4

6. PERMANENT BONDING .................................................................6

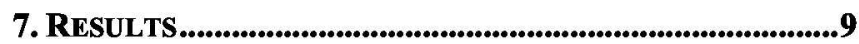

8. ConCLUSIONS.........................................................................11

9. FUTURE WORK .......................................................................11

REFERENCES....................................................................................12

BIOGRAPHY …......................................................................12

\section{INTRODUCTION}

Aligning thin glass segments used for the optics of the International X-Ray Observatory (IXO) poses an interesting challenge. IXO is a project designed at building upon the success of previous X-ray missions such as Chandra and XMM Newton. (For IXO mission background, see [1]). It will have a much larger effective area than any previous $\mathrm{x}$ ray mission with 3.0 square meters at $1.25 \mathrm{keV}$ with an angular resolution of five arc-seconds. The designed double

\footnotetext{
${ }^{1}$ U.S. Government work not protected by U.S. copyright

${ }^{2}$ IEEEAC paper \#1336, Version 2, Updated October 26, 2010
}

reflection focal length of the system is 20 meters (previously mission goals called out 8.4 meters). The work presented in this paper all relates to the 8.4 meter combined focal length because hardware was built for test purposes at 8.4 meters and would require much more effort to change than is worth it because it shouldn't be a significant change. Because IXO is going to operate in the X-ray spectrum, grazing incidence optics are required where the $\mathrm{x}$-ray photons deflect off the mirror at about a 1 degree angle. A Wolter-I type telescope design was selected where the incoming $x$-ray photons graze off of a primary mirror and a secondary mirror at a very small angle to get to the detector. There are several segments nested close together to maximize effective area. The nested mirror segments were selected to be $0.4 \mathrm{~mm}$ thick to conserve mass and maximize collecting area. Meeting the angular resolution requirement of five arc-seconds with such thin glass segments presents a challenge.

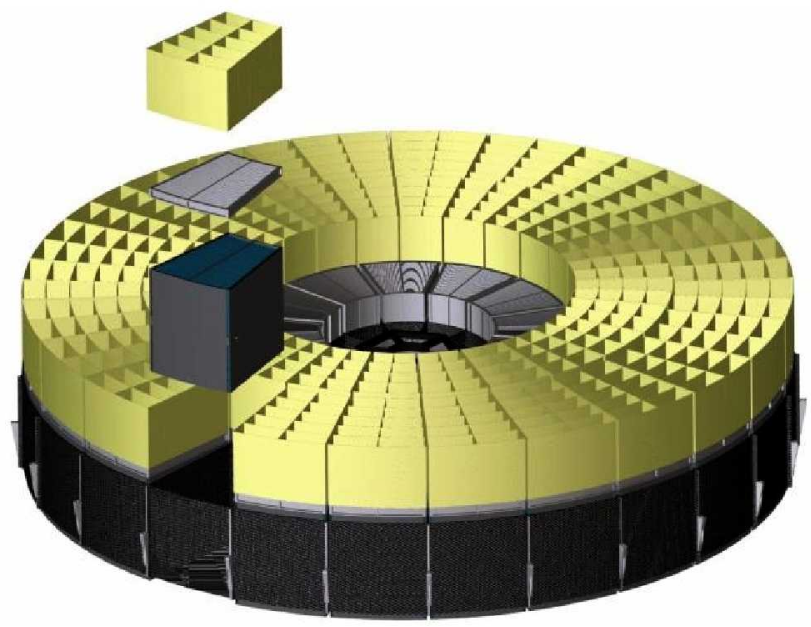

Figure 1 - Modular Flight Mirror Assembly Design

To accommodate all of the mirrors for the telescope, a modular design was conceived. The Flight Mirror Assembly (FMA) will support 60 modules arranged in three rings, 12 inner, 24 middle, and 24 outer as shown by Figure 1 [2]. There will be 200 to 280 mirror segments per module for a total of about fourteen thousand mirror segments. The 
primary and secondary mirrors must be aligned to each other to meet the strict angular resolution requirement. In addition, all of the mirror pairs must focus to the same point within the required angular resolution. The mirror segments being used are made by slumping flat glass onto polished mandrels [5]. The mirror segments are $200 \mathrm{~mm}$ long in the axial direction and have a circumferential span of up to 360 $\mathrm{mm}$. This makes each mirror about the size of a standard piece of 8.5 " by 11 " paper, and about four pieces of paper thick.

There are currently three approaches being developed to solve the challenge of aligning and mounting the mirror segments into a permanent structure. In the first approach, the mirror is adjusted with small high resolution linear actuators to correct for axial and figure errors [3]. This method is being pursued by a team at the Smithsonian Astrophysical Observatory (SAO). The second method involves forcing the mirror segment into a prescribed geometry [4]. This approach is being investigated at the European Space Agency (ESA) and associated industries. The third method is to preserve the fabricated state of the mirror and not introduce any distortion or figure error throughout the alignment and mounting processes. This third method, known as the suspension mount, is being developed at NASA's Goddard Space Flight Center (GSFC) and will be discussed in this paper.

This paper discusses sizes that are sometimes hard to comprehend such as linear measurements of microns and angular measurements of arc-seconds. One micron is $1 \times 10^{-6}$ meters, or conversely, one meter is made up of one million microns. One arc-second is $1 / 3600$ of a degree, there are 60 arc-seconds in one arc-minute and 60 arc-minutes in 1 degree. Although the smallest size visible to the human eye is about $\mathbf{1 0}$ microns, many things discussed in this paper are less than 1 micron and are measured with laser displacement sensors or interferometers. See Table 1 below for sizes of some common small objects.

Table 1. Scale Comparison

\begin{tabular}{|c|c|}
\hline Object & Diameter \\
\hline Human Hair & $\sim 100$ microns \\
\hline $\begin{array}{c}\text { Period at the end } \\
\text { of a sentence }\end{array}$ & $\sim 400$ microns \\
\hline
\end{tabular}

\section{Mirror Segments}

\section{Mirror Segment Background}

The individual mirror segments are slumped from D263 glass onto polished mandrels [5]. The mirror segments are
$200 \mathrm{~mm}$ long in the axial direction and have a circumferential span of up to $360 \mathrm{~mm}$. This makes each mirror about the size of a standard sheet of paper. The mirror segments are $0.4 \mathrm{~mm}$ thick, or about four pieces of paper thick.

The mirrors are grouped into three rings of modules with 12 to 24 modules in each ring, with an average of 240 mirror segments in each module. The combined group of mirror segments, modules, and support structure forms the FMA. The total mass of the FMA is about $1300 \mathrm{~kg}$ [2].

The prime goal of this mission is to be fit $3 \mathrm{~m}^{2}$ of effective area at the soft $\mathrm{x}$-ray band within this mass envelope. Previous high angular resolution x-ray imaging missions such as Chandra and XMM-Newton had much larger mass to area ratios. Per aperture area, Chandra and XMM-Newton required nearly $20,000 \mathrm{~kg}$ and $2,000 \mathrm{~kg}$ per each square meter of aperture, while IXO will be at least an order of magnitude better at about $300 \mathrm{~kg} / \mathrm{m}^{2}$. To accomplish this mass-to-area ratio, IXO uses thin segmented optics instead of full thick shells. This comes at a trade off however, as the thin nature of the segments equals a low stiffness. With a low stiffness, the forming, mounting, and alignment are all a challenge as the thin segments can be easily distorted. Because of the thickness and large mass to area ratio of the Chandra for instance, 0.5 arc-seconds of angular resolution was achieved. Nevertheless, IXO aims to achieve a factor of nearly 3 better angular resolution than that of XMMNewton, and at the same time maintaining a magnitude better mass-to-area ratio.

\section{Preliminary Budget of Error Contributions}

With the best current knowledge of how these mirror segments may be made and form the FMA, an error budget is developed to reach the angular resolution of about 4 arcseconds at the FMA level. The overall mission level requirement is defined at 5 arc-seconds. In Table 2, the allocation of high level error components is listed. The measurements are traditionally reported in Half-PowerDiameter (HPD), where the angular resolution is defined as the angular size within which half the photons were enclosed. The running sum column in Table 2 is simply the root-sum-square difference of the consecutive individual process contributions. Despite the fact that HPD cannot strictly be root-sum-squared, the values of individual components serve as a guide in process development.

As shown in Table 2, a total of 1.26 arc-seconds of error is budgeted to be introduced to the mirror during the temporary mount and permanent bond procedures. This can be further broken down into 0.89 arc-seconds for the temporary mount and 0.89 arc-seconds for the permanent bond. This feeds into the plan to have a final error of 4.14 arc-seconds at the FMA level of assembly which will meet the final 5 arc-second requirement of the mission. 
Table 2. Error budget from fabrication to flight

\begin{tabular}{|c|c|c|c|}
\hline & $\begin{array}{l}\text { Running } \\
\text { Sum } \\
\text { (arcsec) }\end{array}$ & $\begin{array}{c}\text { Individual } \\
\text { Process } \\
\text { Contribution } \\
\text { (arcsec) }\end{array}$ \\
\hline \multirow{3}{*}{ 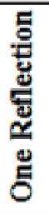 } & Forming Mandrel & 1.47 & 1.47 \\
\hline & $\begin{array}{c}\text { Mirror } \\
\text { Fabrication }\end{array}$ & 2.41 & 1.91 \\
\hline & $\begin{array}{c}\text { Temporary } \\
\text { Mount and } \\
\text { Permanent Bond }\end{array}$ & 2.72 & 1.26 \\
\hline \multirow{2}{*}{ 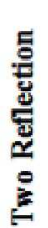 } & Module & 3,86 & 0.34 \\
\hline & FMA & 4.14 & 1.50 \\
\hline
\end{tabular}

The value of 0.89 arc-seconds for the permanent bond procedure is further broken down into individual components. These values are measured using interferomic metrology, Hartmann tests, and some are not yet able to be measured. The Hartmann test mainly measures cone angle variation, which is the largest contributor at this time. The overall rating of the mirror can be achieved from an $\mathrm{x}$-ray test.

\section{TEMPORARY MOUNT}

The temporary mount method being used at NASA's Goddard Space Flight Center (GSFC) is the suspension mount [6]. The idea behind the suspension mount is to preserve the optical figure of the mirror during alignment and bonding into a permanent structure. First, the mirror is hung using four strings to minimize the gravity distortion on the mirror as shown in Figure 2.

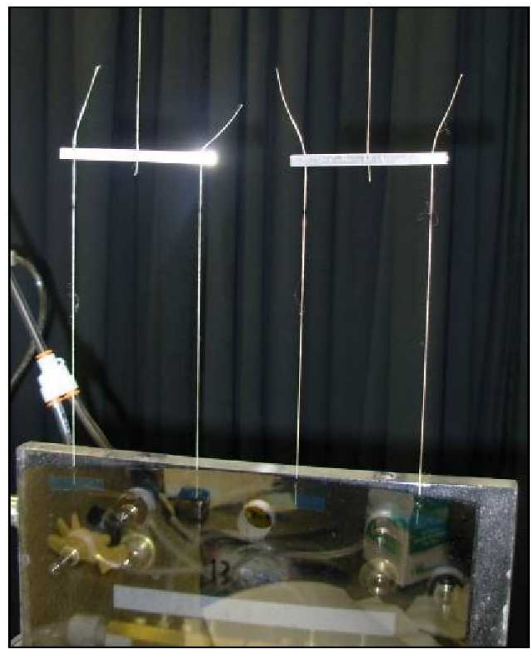

Figure 2 - Four string suspension mount
In order to transport and work with the mirror segment, it needs to be mounted to something. The mirror starts out hanging vertically to minimize gravity distortion and is captured by a fixture called a "strongback". The strongback is a rectangular glass block with a set of pins protruding from its front surface. These pins are set in near-frictionless air bearings so that they apply minimal force when making contact with the mirror.

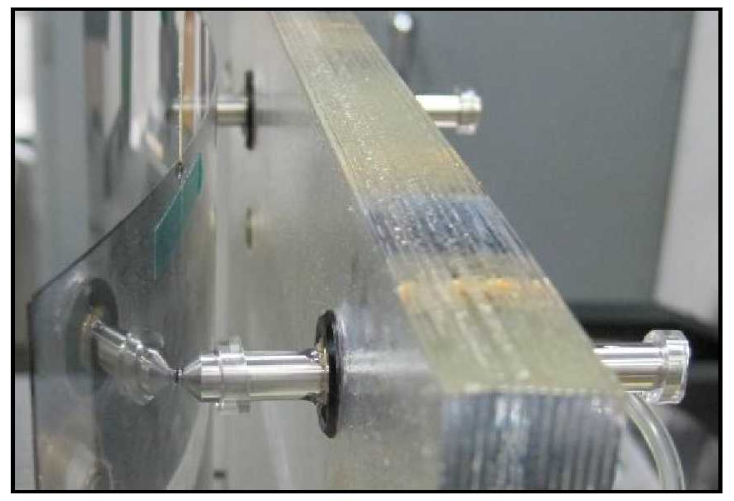

Figure 3 - Pins in air bearings bonded to mirror

The pins are bonded to the back of the mirror as shown in Figure 3, but are still able to float freely to compensate for the mirror swaying or moving. When the mirror settles into its relaxed state, the back of the pins are bonded to strongback, to freeze them in place. This process captures the mirror on the strongback in its hanging state where the distortion is minimized.

\section{Mirror Alignment}

Once the mirror is temporarily bonded, it can be tested for surface quality, and then put into proper alignment. Finite element modeling and practice demonstrate that small adjustments in re-orientation in the gravity field do not distort the mirror figure significantly. The alignment is done with respect to a parallel beam light source.

A six degree of freedom hexapod is used to align the strongback with the temporarily bonded mirror. The hexapod has a repeatability of $\pm 0.5 \mu \mathrm{m}$ in the linear $\mathrm{X}, \mathrm{Y}$, and $Z$ directions (see Figure 4). The controller outputs the absolute position of the hexapod in $\mathrm{X}, \mathrm{Y}, \mathrm{Z}$ coordinates to $0.1 \mu \mathrm{m}$. The rotational position of the hexapod in $\mathrm{U}, \mathrm{V}$, and $\mathrm{W}$ coordinates (see Figure 3) is reported to $10^{-4}$ degrees. Knowing the absolute position of the mirror to this level of accuracy enables calculations to be performed to determine the necessary adjustments for optimizing the image. 


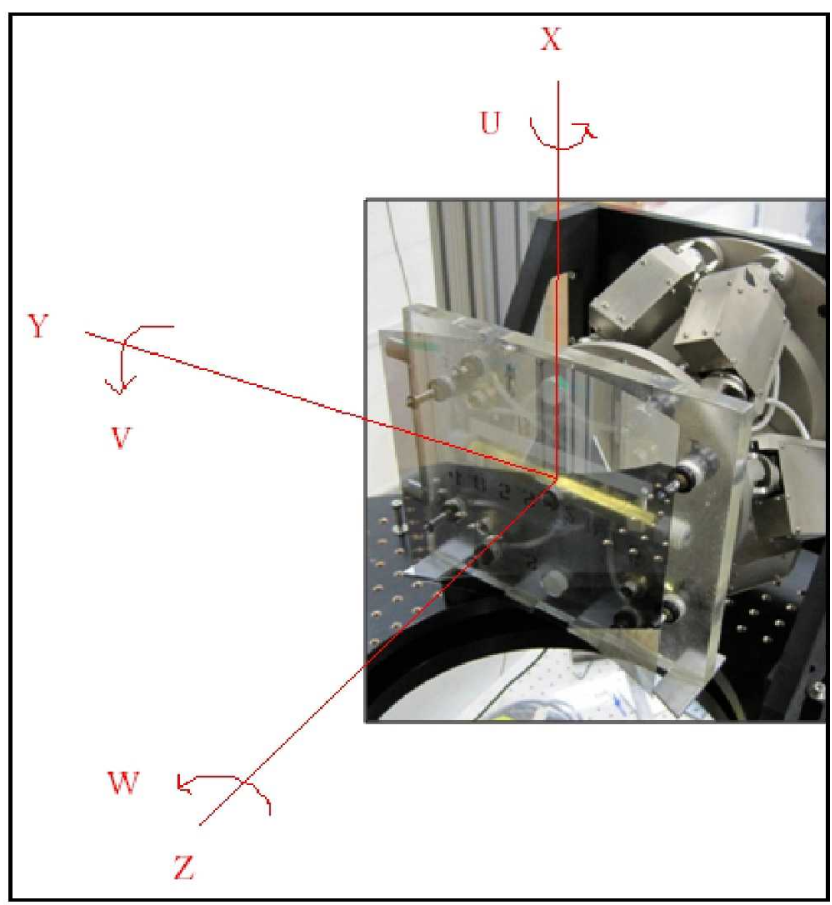

Figure 4 - Hexapod coordinate system

The alignment is mainly adjusted by tilting the mirror in the $\mathrm{V}$ direction (pitch), and by tilting the mirror in the $\mathrm{W}$ direction (yaw). The final way to obtain a better image is to adjust the focal distance by moving the CCD camera at the end of the beam. For data analysis purposes in preliminary trials, it can be desirable to move the CCD camera to get better data to better understand the process. However, when multiple mirror pairs are permanently bonded, they must all focus at the same point since there will only be one detector.

The current mandrels for slumping glass segments were designed for the earlier mission specification of a combined (double reflection) 8.4 meter focal length even though the current mission specification is 20 meters. To achieve this long focal distance of 8.4 meters when the mirror is in a vertical position, a light source is positioned above the mirrors, shone downwards, and then bent 90 degrees using a 45 degree fold mirror so that it is parallel with the optical bench surface. It is then reflected back and forth using flat fold mirrors to achieve the necessary focal length. The light source is a red beam assumed to have a wavelength of 633 $\mathrm{nm}$, which is in the visible light spectrum. Using visible light is a safer way to do testing than shorter wavelengths such as ultraviolet or $\mathrm{x}$-ray. Also, using visible light allows for the path of the light to be traced in order to find the image when large adjustments are made.

The mirror reflection starts as an arc shape (similar to the shadow of the curved mirror) which becomes smaller and smaller until it focuses to a small hourglass shape as shown in Figure 5 (also known as rotated bow-tie). Past the focus, the arc becomes inverted, and grows in size. The focus location determines one component of the alignment. The location of the center of the hourglass itself determines the rest of the alignment. The location of the center of the hourglass is characterized by performing a Hartmann test.

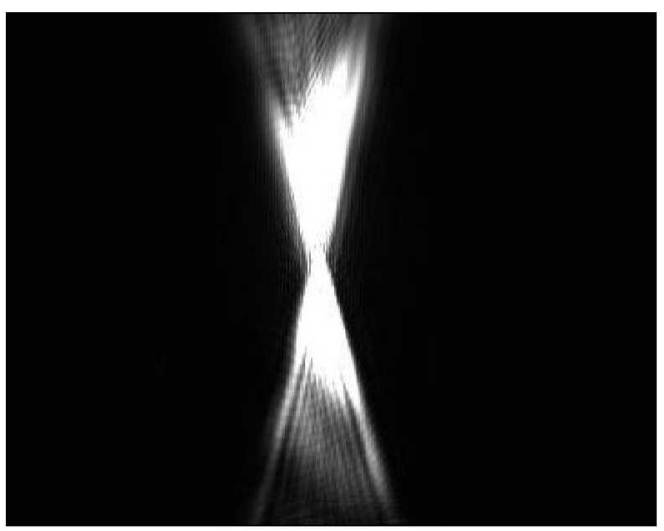

\section{Figure 5 - Image of mirror reflection at focus}

Due to the light source generating a beam of light with a wavelength of about $633 \mathrm{~nm}$, there is a noticeable diffraction effect in the image. Because of the small cone angle of the primary mirror segment, this diffraction effect is large when measuring primary segments which have a focal distance of more than 16 meters.

In order to achieve a good result, the mirror must be tilted at a very specific angle in which the light distribution at the focus is symmetrically distributed across the hourglass shape. A rough estimate of this symmetrical distribution of light can be done by simply looking at the image and correcting. Fine tune adjustments are calculated using the analyzed data. Once a Hartmann test is complete, the general shape of the data set in addition to the magnitude of the errors can be used in conjunction with a set of equations to calculate the necessary adjustments needed for the optimal result. Because the relative position of the mirror between tests is known from the hexapod coordinates, it is possible to quantitatively calculate adjustments. Once a mirror is set-up, the automation of the Hartmann test and data analysis on-site makes it possible to run a test and have results in five minutes. This allows for multiple adjustments to be made and to run iterations to perfect the alignment of the mirror segment. Previous to the use of the hexapod and automated Hartmann analysis, several days were required to align a single mirror segment, where it can now be done in a few hours.

\section{VERIFICATION TESTING}

\section{Test Fundamentals}

A modified Hartmann test is used to test the alignment of the mirror. The test is basically to measure focusing of the mirror by measuring the light ray from sub-apertures of the mirror being tested. In the case of segmented cylinder-like mirror shells such as those of IXO, the simplest sub-aperture 
is an azimuthal slit. This simplifies the test significantly as the test is then a one-dimensional test.

To perform the test, a mask is used to cover the reflection light coming off of the mirror as shown in Figure 6. Only a specific slit of light is allowed to pass through the mask. The mask is then rotated to allow light from different strips of the mirror to be analyzed independently.

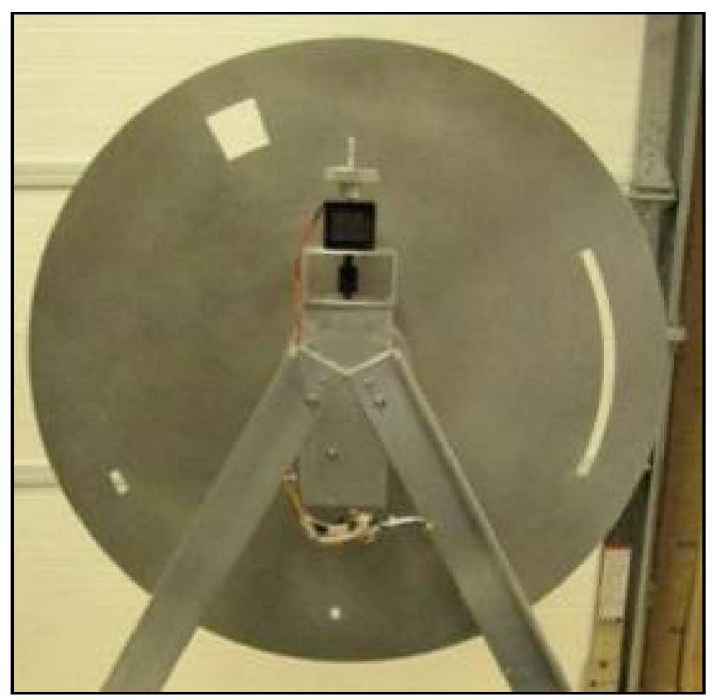

Figure 6 - Hartmann mask

In regards to the hourglass shaped focused image, when only a thin segment of the reflection arc is allowed to pass through the Hartmann mask, a line is displayed. When the lines formed by each stripe of the mirror are put together, they form the hourglass shape as shown in Figure 7.

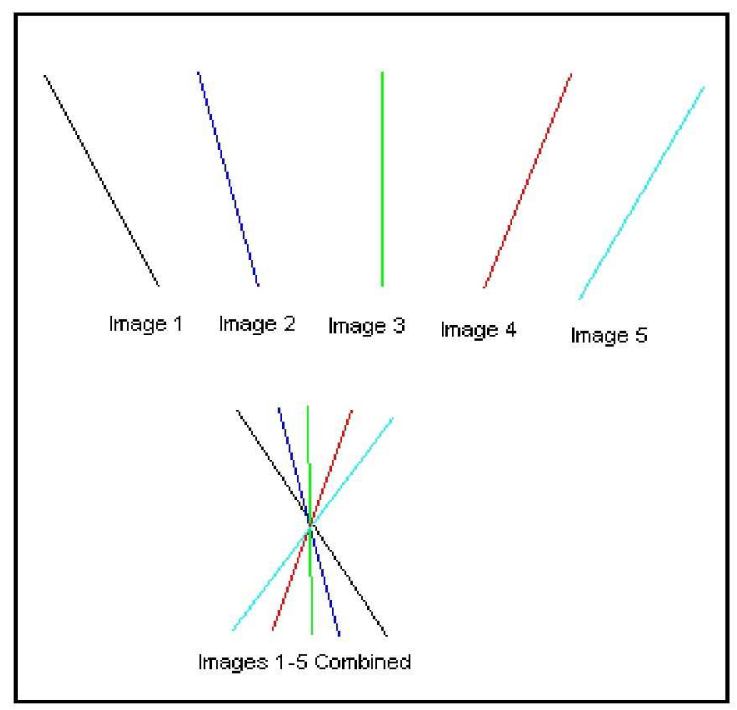

Figure 7 - Combined image explanation

A CCD camera is used to capture an image of each line recording the brightness value of each pixel. The theoretical centroid of the brightness values should be in the center of the hourglass. Therefore the alignment error can be determined from the deviation between the centroids of each of the separate images.

The final outcome of the test is a plot showing the deviation of each centroid location from the average location as shown in Figure 8. Motorized linear stages and a rotational motor have been utilized to automate this entire test.

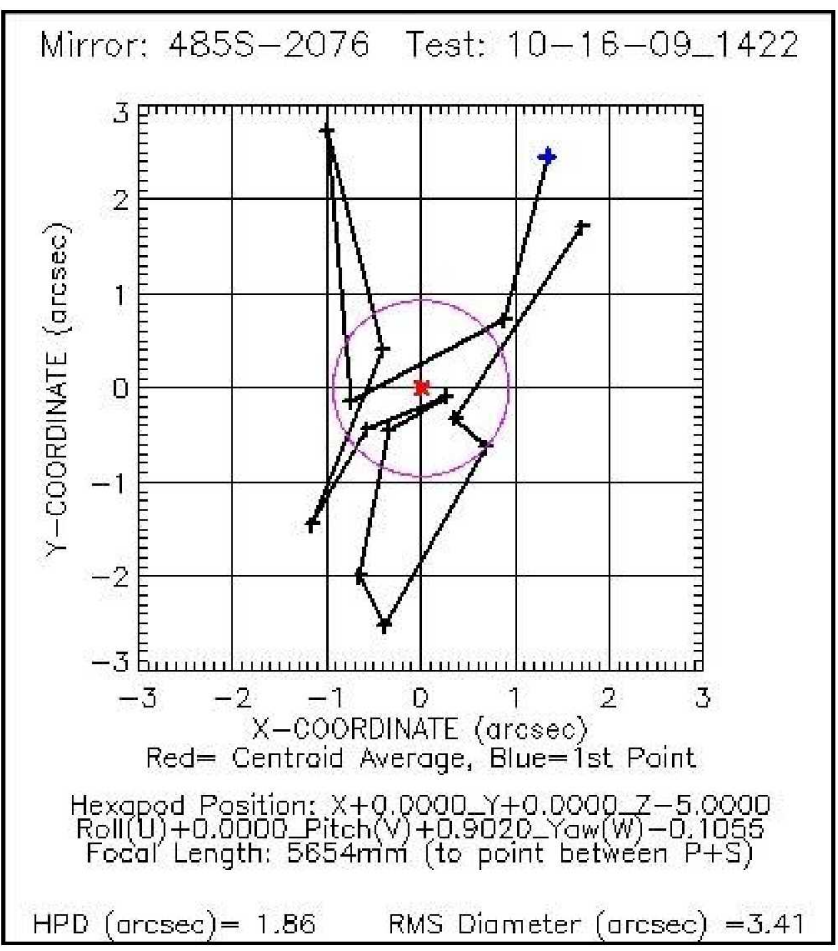

Figure 8 - Sample plot of centroids

The mirror segment alignment parameters are labeled on the graph to track settings used to achieve the image. This helps to understand what changed between trials to improve or degrade the image. The parameters are listed in five major categories. The mirror number is reported to show which mirror is being used. The test number reports the date and time ( 24 hour format) that the test was performed. The hexapod position shows the coordinates that the hexapod was programmed to in order to translate and tilt the mirror to the alignment used during the test. The focal length reports the distance between a middle point between the primary and secondary mirrors and the CCD camera. The HPD and RMS ratings give a value of the spread of the centroids which is used to rate the mirror. The HPD rating of the mirror stands for "half power diameter". It is the diameter of the circle around the average centroid that would contain half of the points. It is signified by the magenta circle in Figure 8. The blue cross signifies the first data point taken, which helps illustrate the shape of the mirror by tracking the individual points with the order they were taken in. The red $x$ indicates the average of all the centroids. 


\section{Data Analysis}

Each image is analyzed independently to find the angle of a line that passes through the sliver of light. This line is represented by a dashed line in Figure 9.

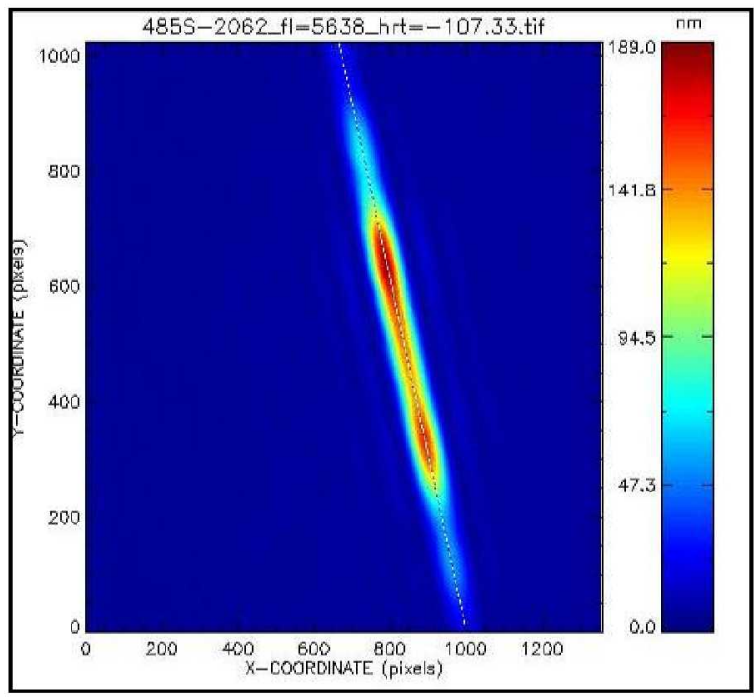

Figure 9 - Analyzed single image from Hartmann test

Once this line has been found, the points along the line are analyzed to compare the brightness of each pixel. The light intensity as a function of focal plane coordinate is shown in Figure 10.

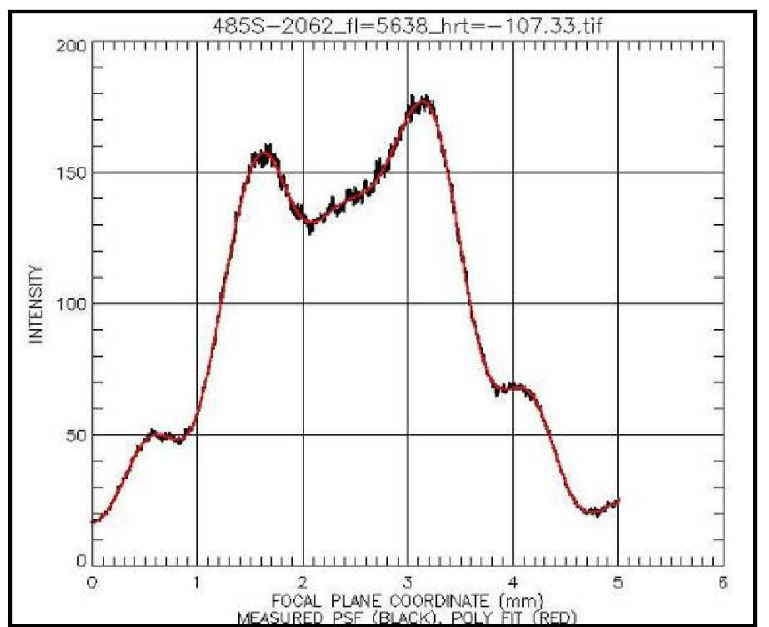

Figure 10 - Light intensity curve along sliver of light

The centroid of the area under this curve is calculated to determine image's centroid. This centroid represents where the center of the hourglass is for that specific image. By comparing the centroids of all of the images, the error rating of the mirror can be determined. Since the diffraction effect does indeed play a large role when using visible light, the final test of the mirror alignment is done using $\mathrm{x}$-ray photons in a vacuum chamber. X-rays have a much shorter wavelength, and the diffraction effect is much smaller.

\section{Permanent Bonding}

Once a mirror segment has been properly aligned, it is permanently bonded into a mock-up of the flight mirror module. For testing purposes, a Mirror Housing Simulator (MHS) is being used to provide bond locations similar to where they would be in the final module design. The MHS is capable of supporting three mirror pairs of different radii. The MHS is constructed of a Ti-15Mo alloy which closely matches the coefficient of thermal expansion (CTE) of D263 glass mirror segments.

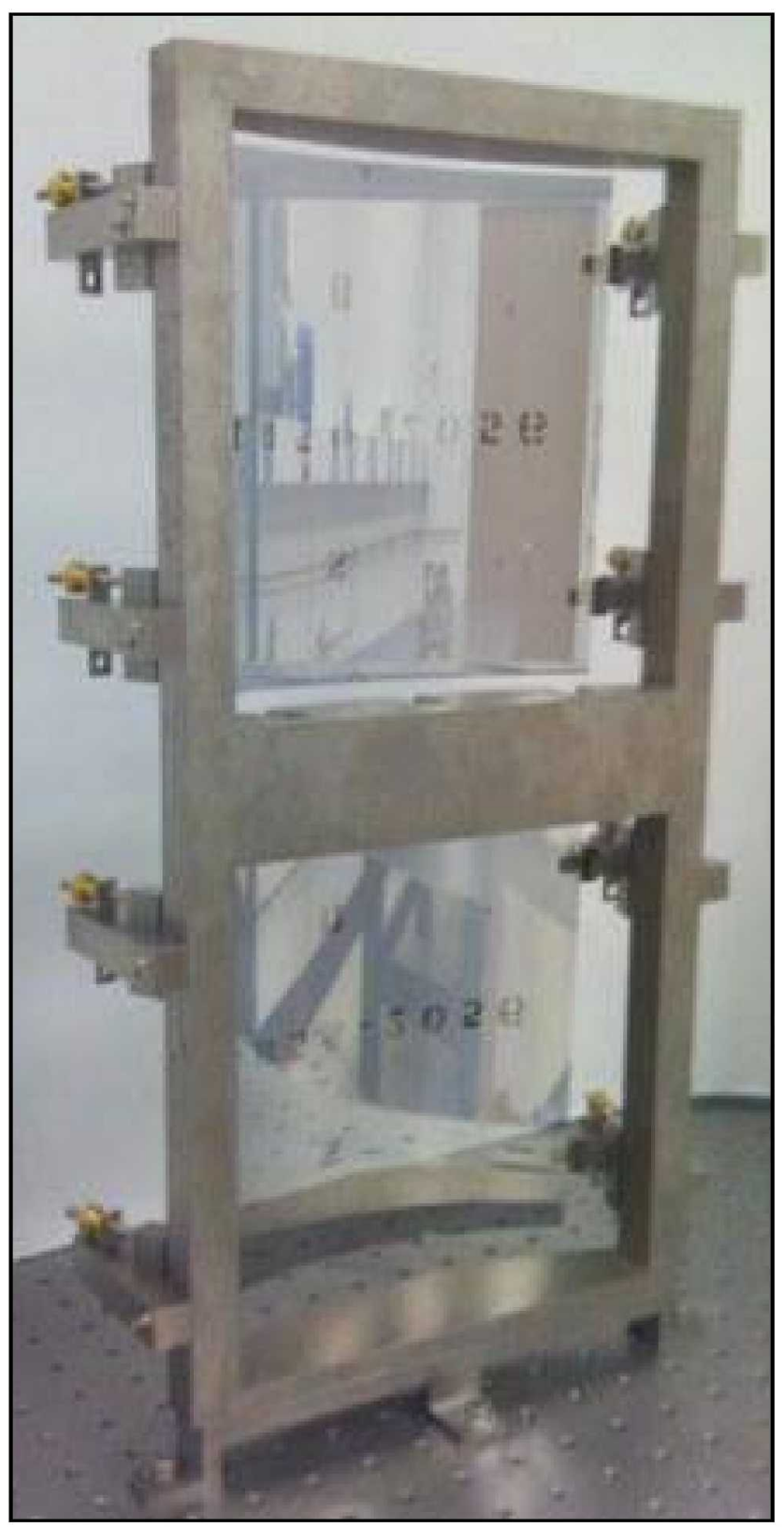

Figure 11 - Mirror Housing Simulator (MHS)

There are twelve rails, six on each side to hold the primary and secondary mirrors. For current testing purposes only the rails at the four corners of each mirror are being used as shown in Figure 11. Small flat tabs slide along the rails into 
position behind the mirror segment as shown in Figure 12 . A miniature stage is used to make the gap size between the tab and the mirror consistent to within a thousandth of an inch for all the tabs. Once in position, the tabs are secured to the rail using epoxy.

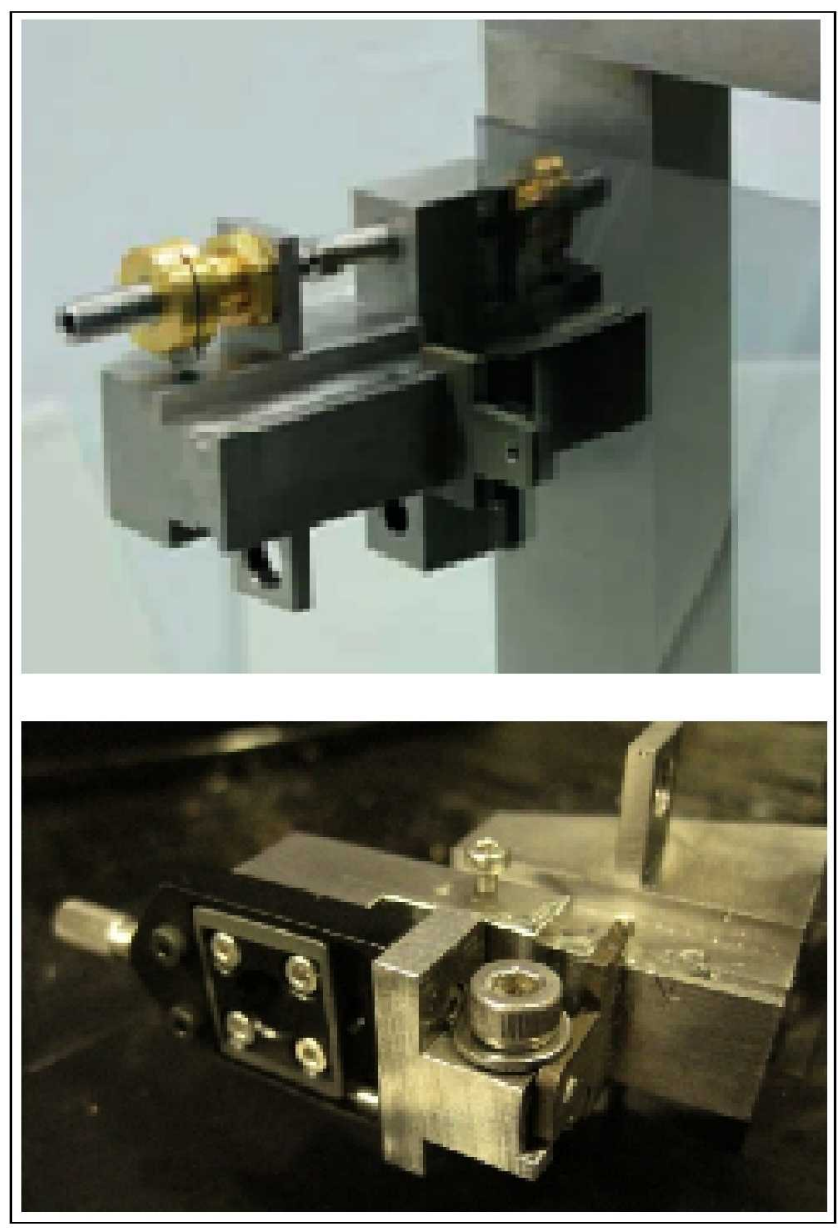

Figure 12 - MHS rail with tab and mirror (top), miniature stage moving tab along rail (bottom)

A closed loop system was designed for the bonding process with a laser displacement sensor (LDS) monitoring the position of the mirror to within 10 nanometers. The data from this LDS is fed into a program that controls the motion of a motorized linear stage called a nano-probe that moves the syringe in sub-micron steps to compensate for epoxy shrinkage and displacements caused by the epoxy making contact with the mirror and the tab. Since the final displacement requirement is less than 0.6 microns of movement, the process has been automated so that it can be performed "hands off" without disturbing the MHS in between bonding tabs. The syringe and nano-probe are mounted to their own tower. Before any bonding occurs, 8 LDS are set up at each of the four bond points for both mirrors. The bond permanent bond hardware is shown in Figure 13 below.
Once the mirror has been bonded to all four tabs, the temporary bonds are broken by twisting the pins, and the strongback is removed. It has been demonstrated that breaking the temporary bonds does not damage the mirror.

A new UV epoxy cure technique was created to bond the mirror to the tab while imposing less than 0.15 microns of displacement. This is the perceived allotment of shift in mirror position that would be allowed under the current error budget scenario for preserving the shape of the mirror for acceptable optical quality. The short term goal is to achieve better than 0.6 microns final displacement, but less than 0.15 microns is the ultimate long term goal. Bonding causes optical distortion due to the shrinkage of epoxy as it cures, so UV cure epoxy and Hysol 9313 have been investigated.

A small high resolution linear actuator with a resolution of $30 \mathrm{~nm}$ is used to move the syringe. The actuator is wired into a closed loop system utilizing a Laser Displacement Sensor (LDS) with a resolution of $10 \mathrm{~nm}$. The actuator oscillates the syringe tip in and out of the tab to move the mirror using the viscous forces from the liquid epoxy. The syringe is oscillated until the mirror has reached the desired offset position. This offset is determined by how much epoxy shrinkage will occur during the cure using the UV light. The epoxy is then cured, bringing the final displacement to zero. The setup for this process is depicted in Figure 13.

As shown in Figure 13, the Laser Displacement Sensor (LDS) monitors the mirror while the epoxy syringe is brought in from the other side to bond the mirror to the tab. 

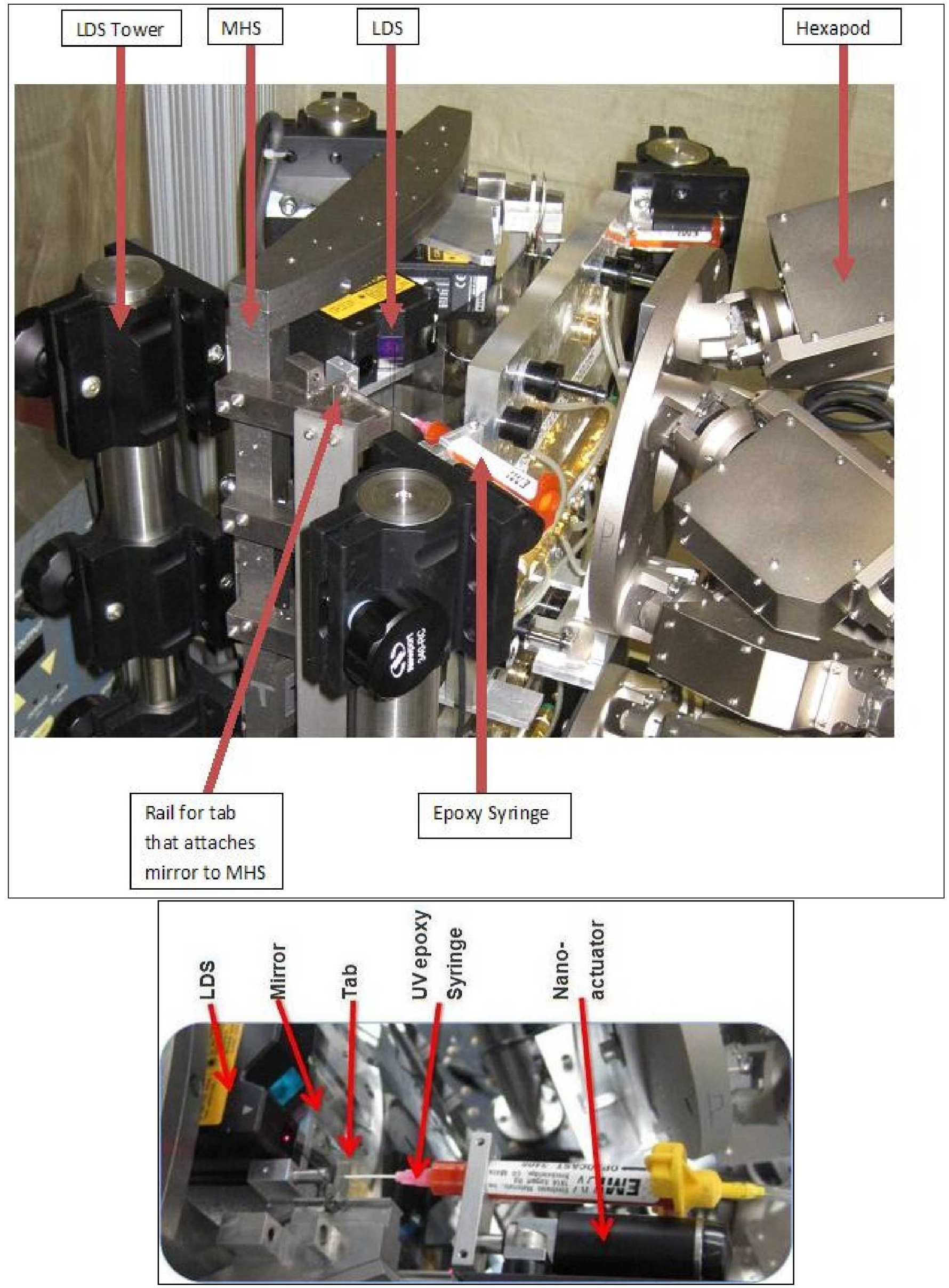

Figure 13- Permanent Bonding Setup 


\section{RESULTS}

Permanent Bond Cycle 2: May 2010

The mirror pair 485C-2111 was bonded into the MHS without adding noticeable distortion. As shown in Table 3, the results from the Hartmann tests taken before and after bonding were within the error of the measurement. This implies that negligible distortion was added during the bonding process. However with the suspension mount method, the fundamental idea is to preserve the shape of the mirror, not improve it. So if distortions are added during fabrication or temporary bonding of the mirror, nothing can be done to fix those distortions in the permanent bond process. Unfortunately, the more distortion added during the initial processes, the higher the error rating of the mirror at the alignment stage. Since errors are combined using an RSS method, the larger the initial error, the less sensitive the process is to detecting future distortions.

Table 3. Alignment of mirror pair 485C-2111, May 2010

\begin{tabular}{|c|c|}
\hline Temporary Bond Alignment & $11.4+/-1.25$ arc-seconds \\
\hline Permanent Bond Alignment & $10.9+/-1.25$ arc-seconds \\
\hline
\end{tabular}

In the bonding of mirror pair 485C-2111, the mirror movements were all less than or equal to 0.6 microns excluding one outlier due to a human error of monitoring the wrong sensor. FEA analyses have shown that a standard deviation in permanent bond displacement of 0.6 microns would yield a 4" HPD error which would meet the allocation allowed for the TRL 4 goal currently being pursued. The individual results for each tab bonded during the most recent trial are shown in Table 4. In all of the trials except for one that was omitted due to an error in measurement, the permanent bond successfully meets the goal of 0.6 microns. This LDS data corresponds well to the previous table which showed that the Hartmann results from the mirror segments before they were bonded, and after they were bonded and transferred were the same within the noise of the test. In future trials, a better temporary mount will increase the measurement sensitivity, and the permanent bond technique can be fully proven out. By ensuring the mirror pair does not change to within 4 arc-seconds, the mirror pair will be on track to meeting the requirements for TRL 4.

Table 4. Bonding of mirror pair 485C-2111, May 2010

\begin{tabular}{|c|c|c|c|c|c|c|}
\hline & Tab 1 & Tab 2 & Tab 3 & Tab 4 & Avg & $\begin{array}{c}\text { Std } \\
\text { Dev }\end{array}$ \\
\hline Secondary & -0.6 & -0.3 & -0.2 & 0.0 & \multirow{2}{*}{0.27} & \multirow{2}{*}{0.24} \\
\cline { 1 - 4 } Primary & 0.6 & 0.1 & 0.1 & ${ }^{*}$ N/A & & \\
\cline { 1 - 3 } \\
*During bonding of tab 4, the wrong LDS was used and therefore the \\
movement of the mirror was not recorded accurately.
\end{tabular}

To reach flight qualifications, a permanent bond displacement of less than 0.15 microns will need to be achieved in order to yield less than 1" HPD. A repeatable process to achieve a permanent bond displacement standard deviation of less than 0.15 microns from zero has been demonstrated in a side experiment, but has yet to be fully proven on actual mirror segments.

\section{Permanent Bond Cycle 3: July 2010}

The mirror pair 485C-2111 was bonded again in July 2010 . The overall result was not great, but several lessons were learned. As shown in Table 5, the alignment of the mirror pair was 10.83 arc-seconds before bonding, and increased to 26.72 arc-seconds after bonding. The distortion caused during bonding was believed to be caused by environmental temperature and bond gap size.

Table 5. Alignment of mirror pair 485C-2111, May 2010

\begin{tabular}{|c|c|}
\hline Temporary Bond Alignment & $10.83+/-1.25$ arc-seconds \\
\hline Permanent Bond Alignment & $26.72+/-1.25$ arc-seconds \\
\hline
\end{tabular}

Since it was known that the temperature in the lab was most stable at night, the bonding was started at 4:00 AM. The intent was to start at Midnight and be done by $8: 00 \mathrm{AM}$, but starting late pushed the rest of the process into the next morning and afternoon where the temperature started to rise, distorting the mirrors at the sub-micron level in the process. During the bonding of the first mirror, the temperature was held to within $+/-0.1^{\circ} \mathrm{C}$. Since the second mirror had to be adjusted to compensate for the first mirror bond distortion, the second mirror was not bonded until 1:00 PM and the temperature increased almost $2^{\circ} \mathrm{C}$ during the bonding of the second mirror. Since the CTE properties of the MHS and the glass of the mirrors are not exactly matched, different components of the system expand/contract at different rates. This was one of the causes of distortion that led to the result of 26.72 arc-seconds post bond. The second cause of distortion was that some of the individual permanent bonds were greater than 1 micron as shown by Table 6 .

Table 6. Bonding of mirror pair 485C-2111, July 2010

\begin{tabular}{|c|c|c|c|c|c|c|}
\hline \multicolumn{7}{|c|}{ Mirror movement during bonding $(\mu \mathrm{m})$} \\
\hline & Tab 1 & Tab 2 & Tab 3 & Tab 4 & Avg & $\begin{array}{c}\text { Std } \\
\text { Dev }\end{array}$ \\
\hline Secondary & -2.8 & 0.8 & 0.8 & 2.5 & \multirow{2}{*}{0.27} & \multirow{2}{*}{0.24} \\
\hline Primary & 0.0 & 1.5 & 0.5 & 0.9 & & \\
\hline \multicolumn{7}{|c|}{$\begin{array}{l}\text { *During bonding of tab } 4 \text {, the wrong laser displacement sensor was } \\
\text { used and therefore the movement of the mirror was not recorded } \\
\text { accurately. }\end{array}$} \\
\hline
\end{tabular}

The designed gap size between the mirror and the tab during a permanent bond is 10 thousandths of an inch. Of the eight permanent bonds during permanent bond cycle 3 , five of 
them were the correct 10 thousandths. The three that were different were all above 15 thousandths. Those three larger gap sizes also happen to be the three distortions that were larger than 1 micron (Secondary Tab 1 and 4, Primary Tab 2). It is believed that a larger gap size correlates to a larger permanent bond distortion. A side experiment has been done to confirm this. The side experiment actually points towards and optimum gap size of around four thousandths of an inch.

Even though the final result was not very successful (26.7 arc-seconds), several lessons were learned from this bond cycle. Temperature sensitivity was exposed as a driving factor in affecting the mirror alignment during the bonding. Bond gap size was also identified as important and the future procedure was tweaked to improve upon this. After this bond cycle a new UV cure machine was purchased that used a LED head which output less heat and caused less movement of the mirror during curing.

Permanent Bond Cycle 4: September 2010

The mirror pair 489C-2058 was bonded in September 2010. The overall result was the best ever achieved by the project, and the mirror were taken to $\mathrm{x}$-ray testing for a more comprehensive analysis. The best alignment achieved ever by the project was achieved by these two mirrors on the hexapods with a result of 1.1 arc-seconds HPD, 1.9 arcseconds RMS diameter. The mirrors changed slightly after that result, and were at 4.0 arc-seconds RMS diameter at the start of permanent bonding. After bonding and transferring the mirrors to the MHS, the final alignment result was 10.7 arc-seconds RMS diameter, 9.8 arc-seconds HPD.

Table 7. Alignment of pair 489C-2058, September 2010

\begin{tabular}{|l|c|}
\hline Temporary Bond Alignment & $4.0+/-0.3$ arc-seconds \\
\hline Permanent Bond Alignment & $10.7+/-0.3$ arc-seconds \\
\hline
\end{tabular}

All eight of the permanent bonds were less than a micron so the individual point distortion was small. A new 300 step checklist was used to make sure no steps were overlooked or performed incorrectly. There were some unforeseen issues however such as cutting the syringe tip with a hot blade, and fastening a UV wand-holder tower to the base plate. A hot blade was used to cut the syringe tip after bonding, but after four bonds, it was determined that the heat radiating from the tip of the hot blade was distorting the mirror and it was not used for bonding the second mirror. A new LED UV wand was used for this cycle which required a new tower to hold the wand in place while curing the epoxy. This tower was bolted to the base plate. It was later revealed that the act of tightening the screw that held the tower down on the base plate to prevent tipping over was distorting the base plate. When the screw was tightened, the whole base plate warped on the sub-micron level, which caused the MHS to tilt slightly, which changed the alignment of the already bonded primary mirror with respect to the secondary mirror that was being bonded.

\section{X-Ray Testing Cycle 1: October 2010}

All of the previous mirror alignment results are produced by optical light Hartmann tests which only rates one type of error seen by the mirror. Performing an x-ray test grazing photons off of the mirrors into a CCD detector is the only way of reporting a comprehensive result. Since the mirror pair bonded in September 2010 seemed to have a good result, they were taken to $\mathrm{x}$-ray testing. They were put into a four foot diameter vacuum chamber and pumped down to less than $1 \mathrm{mT}$ Torr at room temperature of $20.4^{\circ} \mathrm{C}$ as shown in Figure 14. A $4.5 \mathrm{keV}$ titanium source was placed 600 meters downstream from the mirrors and a CCD detector is placed at the combined focal distance past the mirrors. There is an eight meter section of pipe that connects the chamber to the CCD.

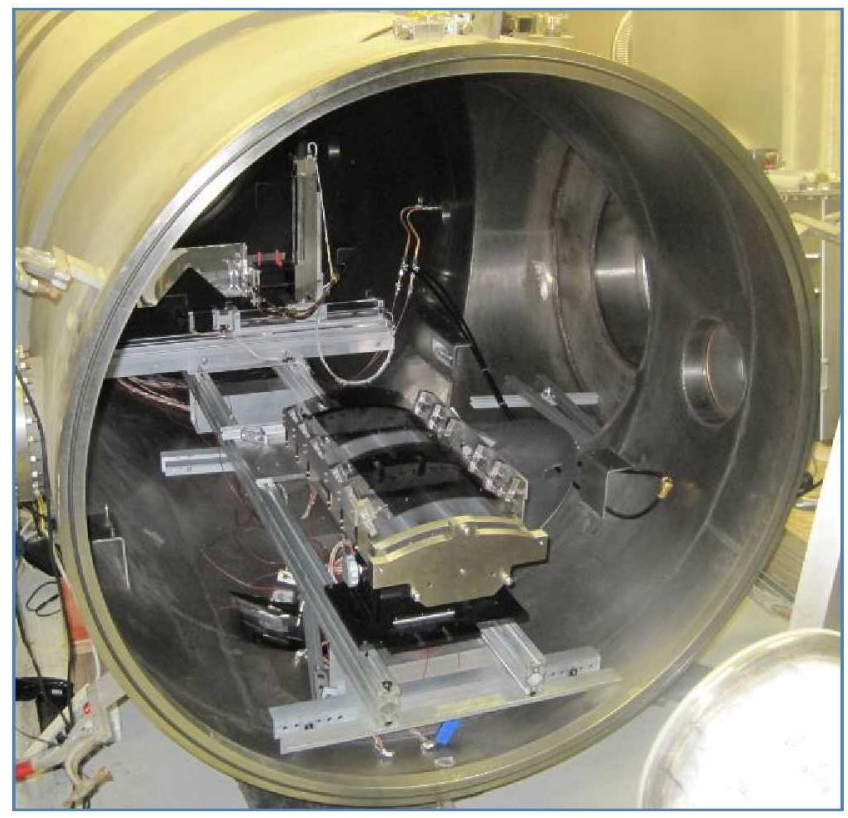

Figure 14 - MHS loaded into vacuum chamber

There were several milestones for the x-ray testing of this mirror pair. This was the first time two mirrors had been permanently bonded to a single fixed structure and x-ray tested. Previously, each mirror was bonded to its own structure and the mirrors could be adjusted relative to each other during the $\mathrm{x}$-ray test to optimize the result.

The x-ray testing on the $489 \mathrm{C}-2058$ mirrors from permanent bond cycle four produced the best results in the history of the IXO project. The best X-ray image was measured at 9.7 arc-seconds HPD for the central 34 degrees of the mirrors. The results are shown in Table 8 . The project goal for TRL 4 is to complete three $x$-ray tests under 15 arc-seconds, so this counts as one of the three and once it is repeated, TRL 4 will be met. 
Table 8. X-ray results of mirror pair 489C-2058, October 2010

\begin{tabular}{|c|c|c|}
\hline \multicolumn{3}{|c|}{$\begin{array}{c}\text { X-ray test results } \\
\text { (HPD in arc-seconds) }\end{array}$} \\
\hline Portion of mirror tested & Average & $\begin{array}{c}\text { Standard } \\
\text { Deviation }\end{array}$ \\
\hline $\begin{array}{c}\text { Central 34 degrees } \\
\text { (10 measurements) }\end{array}$ & 11.3 & 1.0 \\
\hline $\begin{array}{c}\text { Full mirror } \\
\text { (5 measurements) }\end{array}$ & 12.7 & 0.9 \\
\hline
\end{tabular}

During the $\mathrm{x}$-ray test, the temperature was cycled from a test temperature of $20.4^{\circ} \mathrm{C}$ up to $27^{\circ} \mathrm{C}$ down to $17^{\circ} \mathrm{C}$, and back to the test temperature. As expected, the HPD result was considerably worse at the higher and lower temperatures. The important result of this temperature cycle sideexperiment was that the mirror returned to its original shape and the results did not change. The vacuum chamber was also vented to atmospheric pressure halfway through the test. The chamber was opened up and the mirrors were removed from their kinematic mount and then placed back in. After pumping the vacuum chamber back down to less than $1 \mathrm{mTorr}$, the same results were achieved. This helps prove the robustness of the test to outside factors such as temperature, placement inside the chamber, and the pumpdown, vent-up processes.

\section{Conclusions}

The mission requirements for IXO of large effective area and high angular resolution do not leave much room for error in the alignment and mounting of thin mirror segments. However, this has driven the design of new hardware and procedures to accommodate these challenges. Achievements in mirror fabrication and temporary mounting have provided better mirror substrates to be aligned and bonded. The use of sensors and actuators to control the bonding process has led to advances in deformation control to the sub-micron level. These changes have all been validated by a record-breaking x-ray test result below 10 arc-seconds HPD of a pair of mirrors permanently bonded to a single fixed structure.

Because of the modular design of the FMA this work should apply directly to the other segments to help make this mission a reality. Future work includes performing another test cycle to demonstrate repeatability of the process. Once this has been demonstrated, work will shift towards coaligning multiple pairs of mirrors to the same nominal focal point.

\section{FUTURE Work}

There is a lot of future work planned including more $\mathrm{x}$-ray tests for the current mirrors, transitioning to new mirrors, and trying a new procedure. The current procedure will be repeated to prove that it is a repeatable process and not just a lucky test. Preferably the same mirrors will be used, but if not a similar pair of mirrors is available for testing. Some procedural changes for the current method will also be explored such as a smaller bond gap size, different epoxies, and quicker turn-around rate to reduce environmental changes.

The current mirrors being tested are 50 degrees in angular span but new 30 degree mirrors are being manufactured to a much better angular resolution. The new mirrors are more promising for future $\mathrm{x}$-ray tests since they start at a better quality before temporary or permanent bond distortions are added. New mounting hardware is currently under development to take advantage of these better mirror segments.

In addition to the changes to the current procedure, a whole new method of permanently bonding the mirrors is being developed. Instead of mounting a flat tab to the back surface of the mirrors, pins would be bonded to the side edges of the mirror where it is naturally stiffer. This "edge-bonding" method is showing promise in early trials, and if successful more time and effort may be transitioned from the current tab approach to this new side pin approach. 


\section{REFERENCES}

[1] "The International X-ray Observatory", Nick White, et al., in Proceedings of "X-ray astronomy 2009 Present status, multi-wavelength approach and future perspectives", Astro-Ph, arXiv:1001.2843.

[2]R. McClelland and T. Carnahan, "Design and Analysis of the International X-Ray Observatory Mirror Modules," 2010 IEEE Aerospace Conference Proceedings, March 7$14,2010$.

[3] W. Podgorski, D. Caldwell, M. Freeman, et al., "A Mounting and Alignment Approach for Constellation-X Mirror Segments," Proc. of SPIE, 7011, 701112, 2008.

[4] M. Beijersbergen, S. Kraft, R. Gunther, et al, "Silicon Pore Optics: Novel Lightweight High-Resolution X-Ray Optics Developed for XEUS," Proc. of SPIE, Vol. 5488, 868, 2004.

[5] W. Zhang, et al., "IXO Mirror Technology Development," Proc. of SPIE, Vol. 7437, 2009.

[6]K-W. Chan, W. Zhang, T. Saha, et al., "An Approach for Alignment, Mounting and Integration of IXO Mirror Segments," Proc. of SPIE, Vol. 7437, 2009.

\section{BIOGRAPHY}

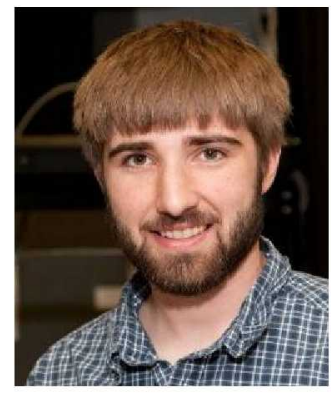

Tyler Evans is a Mechanical Systems Engineer at SGT Inc. currently working on the alignment and bonding of mirror segments for IXO at the NASA Goddard Space Flight Center. He received a B.S. in Mechanical Engineering from Purdue University.

Scientist at the University of Maryland, Baltimore County currently working on development of the $x$-ray mirror assembly for IXO at the NASA Goddard Space Flight Center. His previous experience includes work on the x-ray telescopes for the Japan-U.S. mission Suzaku. Prior to Suzaku, he was a member of the mirror team which developed the X-Ray Telescopes for the original Astro-E and the balloon flight program InFOCuS. He was a member of the Science Working Group for both Astro-E and Suzaku. He received a B.S. in Physics from the Chinese University of Hong Kong, and a M.S. and Ph.D. in Physics from the University of California, San Diego.

Timo Saha is a physicist in the Optics Branch at NASA/Goddard Space Flight Center. He is working on design and development of the $x$-ray mirror assembly for the International $X$-ray Observatory. His previous experience includes work on design and optical modeling of $X$-ray, EUV, and FUV optical systems. He received a B.S. and M.S. in Physics from the University of Turku, Finland, and a Ph.D. in Physics from St. Louis University in St. Louis, Missouri. 